\title{
Arrays of EAP micro-actuators for single-cell stretching applications
}

\author{
S. Akbari*, M. Niklaus, H. Shea \\ Ecole Polytechnique Fédérale de Lausanne (EPFL), Rue Jaquet-Droz 1, CP 526, CH-2002 \\ Neuchâtel, Switzerland
}

\begin{abstract}
Mechanical stimuli are critical for the development and maintenance of most tissues such as muscles, cartilage, bones and blood vessels. The commercially available cell culture systems replicating the in vivo environment are typically based on simple membrane cell-stretching equipment, which can only measure the average response of large colonies of cells over areas of greater than one $\mathrm{cm}^{2}$. We present here the conceptual design and the complete fabrication process of an array of 128 Electro-Active Polymer (EAP) micro-actuators which are uni-axially stretched and hence used to impose unidirectional strain on single cells, make it feasible to do experiments on the cytomechanics of individual cells. The Finite Element Method is employed to study the effect of different design parameters on achievable strain, leading to the optimized design. Compliant gold electrodes are deposited by low-energy ion implantation on both sides of a PDMS membrane, as this technique allows making electrodes that support large strain with minimal stiffening of the elastomer. The membrane is bonded to a rigid support, leading to an array of $100 \times 100 \mu \mathrm{m}^{2}$ EAP actuators.
\end{abstract}

Keywords: Dielectric elastomer actuators, Micro-actuator, single-cell stretcher, low energy ion implantation

\section{INTRODUCTION}

\subsection{Dielectric Elastomer Actuators (DEA):}

Dielectric elastomer actuators combine large displacements (up to several hundred percent strain) and high actuation pressure per unit mass (of order $10^{3} \mathrm{~Pa}^{3} \mathrm{~m}^{3} / \mathrm{kg}$ ) with low energy consumption ( $\mu \mathrm{W}$ for full unloaded displacement), only dissipating power when moving, and hence generating negligible heating ${ }^{[1]}$. In addition, since the electrostatic force scales as the inverse of the dielectric thickness, DEAPs are excellent candidates for miniaturization. Microactuators based on EAP enable a broad new range of applications for which large strains and forces are desirable, as their strainforce capabilities are not matched by any standard microactuators classes. The main disadvantage of EAPs is the high voltage required for actuation, of order $1 \mathrm{kV}$.

Dielectric elastomer actuators (DEA) consist of an elastomer sandwiched between two compliant electrodes as shown schematically in Figure 1. When a voltage is applied to the electrodes, an electrostatic pressure $\mathrm{P}$ is generated $P=-\varepsilon_{0} \varepsilon_{r} E^{2}$, where $\varepsilon_{0}$ is the vacuum permittivity, $\varepsilon_{\mathrm{r}}$ is the relative permittivity and $\mathrm{E}$ is the applied electric field. The pressure adds compressive stress to the membrane. Under free boundary conditions, the film area expands since volume is conserved. A wide variety of actuators and sensors can be made depending on the choice of boundary conditions to promote stretching, buckling, curling, etc.

\subsection{Compliant electrodes by low energy metal ion implantation}

As exposed above, applying a voltage between the electrodes of a DEA can lead to large area expansion of the elastomer, requiring that its electrodes also be able to sustain the same strain without damage and losing conductivity. A large variety of electrode materials have been explored such as thin metal films, metallic paints, dry carbon powder or carbon nanotube thin films (for instance ref [1] for a review). Thin metallic films provide good electrical conductivity but high stiffness and with maximum strain limited to $1 \%$ to $3 \%$, while carbon grease electrodes are good conductors even at high strains, and very soft, making them the most commonly used electrodes for macro-scale applications.

*s.akbari@epfl.ch; phone +41 32720 5460; fax +41 32720 5754; http://lmts.epfl.ch

Electroactive Polymer Actuators and Devices (EAPAD) 2010, edited by Yoseph Bar-Cohen,

Proc. of SPIE Vol. 7642, 76420H - (c) 2010 SPIE · CCC code: 0277-786X/10/\$18 - doi: 10.1117/12.847125

Proc. of SPIE Vol. $764276420 \mathrm{H}-1$ 
When scaling down to micro-actuators, carbon powder or carbon grease electrodes are of less interest since they are not compatible with cleanroom facilities and are difficult to pattern with $\mu \mathrm{m}$ resolution. Our laboratory has developed a technique using low-energy ion implantation to create highly deformable electrodes on polydimethylsiloxane (PDMS) ${ }^{[2]-}$

[5]. Implantation leads to the creation of nanometer-size clusters in the first $50 \mathrm{~nm}$ below the surface. When the elastomer is stretched, these small clusters can move relative to one another, maintaining electrical conduction at strains of up to $175 \%{ }^{[5]}$.
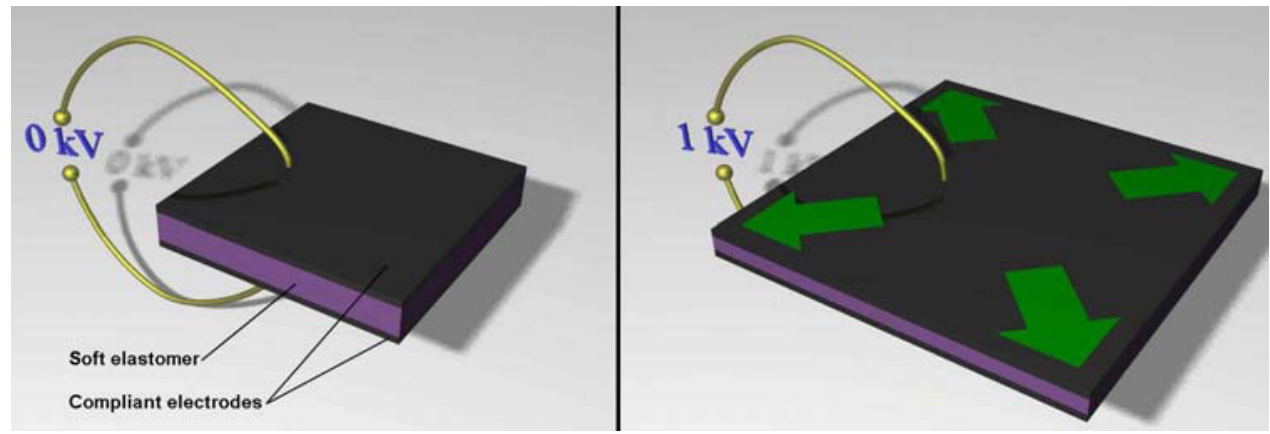

Figure 1-When a voltage is applied to the electrodes; the electrostatic pressure squeezes the elastomer (right side) while being incompressible stretching the structure in the case of free boundary conditions ${ }^{[6]}$

We use a plasma-based implantation technique called filtered cathodic vacuum arc ${ }^{[7]}$, which allows to obtain high ion flux at low energies $(0.2-10 \mathrm{keV})$ : for gold implantation, 3 minutes are sufficient to obtain a surface resistance below 1 $\mathrm{k} \Omega$ per square on our system. Our experimental FCVA implanter has a beam size of $1 \mathrm{~cm}^{2}$ and operates in a pulsed mode (Figure 2).
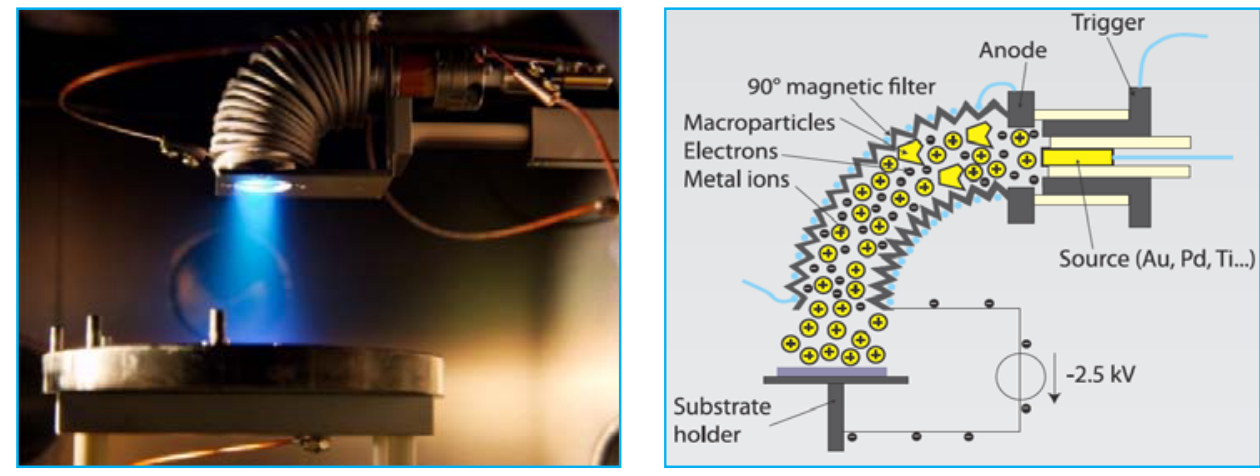

Figure 2- right: Picture of our FCVA implanter during a pulse. Left: Schematic representation of our FCVA implanter. A high voltage impulsion on the trigger electrode initiates the main arc between the source and the anode. The electrons, ions and macroparticles produced by the arc enter the filter which traps the unwanted macroparticles. The substrate holder is negatively biased to accelerate the positive ions through the plasma sheath ${ }^{[2]}$.

The key point of ion implanted electrodes resides in the fact that implantation does not form a continuous polycrystalline film in which grains are attached to each other at the grain boundaries, thus forming a rigid film capable of only a few percent strain before breaking. Instead, ion implantation leads to the formation of small size (2-20 nm) clusters in the polymer matrix (Figure 3). These clusters can touch each other (thus providing a conducting path) without forming a strong mechanical bond. The absence of a strong bond between clusters allows them to slide relative to each other. This leads to a reduced impact on the stiffening (increase of Young's modulus) of the PDMS due to the inclusion of the metallic particles, and an ability to withstand high strains before losing electrical conduction. 


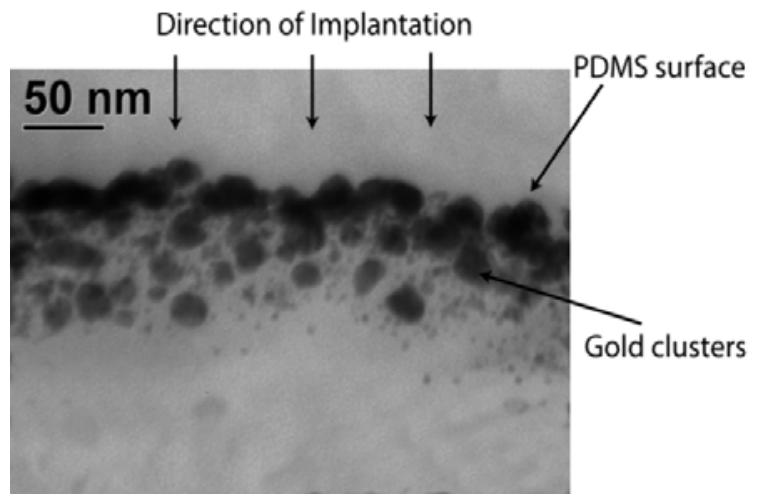

Figure 3- The implantation leads to the creation of nanometer-size clusters which extends from the surface of the PDMS down to approximately $50 \mathrm{~nm}{ }^{[8]}$. The clusters slide relative to one another, allowing conduction at large strains, but much less stiffening than a continuous metallic thin film.

\subsection{Mechanical stimulation of cells}

It is of great clinical importance to investigate the response of body cells to mechanical strain, which is ubiquitous arising from bone elongation, compression within joints, or dynamic mechanical stimulation resulting from muscular activity. It is now clear that cells modulate their behavior and developmental programs in response to mechanical stresses present in the organism. Extracellular forces are transduced across the cell membrane to affect intracellular biochemical events such as proliferation, differentiation, alignment and cell to cell calcium signaling ${ }^{\text {[9]-[14] }}$. The process by which cells convert mechanical signals into biochemical responses is known as mechanotransduction.

Due to complexity of in vivo environment, the systematic study of cell mechanotransduction is relied on cell culture systems designed to replicate the in vivo situations. Varieties of stretchable cell culture substrate systems have been developed to apply controllable mechanical strain to cells in vitro. The majority of devices consist of cells cultured on a simple membrane multiaxially distended either by vacuum (e.g., those made by Flexcell International corp.) or mechanical motor, or uniaxially stretched where there is a single axis of uniform tensile strain with a small magnitude of compression as reviewed by Brown ${ }^{[15]}$.

Employing the above mentioned devices, strain of up to $20 \%$ at frequencies of order $1 \mathrm{~Hz}$ is achievable, but the areas stretched are often in the range of centimeters, much too large to monitor single-cell responses; instead investigators often measure the averaged response of hundreds of thousands of cells that, by synchronizing their communal behavior, make it very difficult to analyze the first stages of cell differentiation. Exactly how the cell is able to decipher subtle changes in common factors to evoke distinct responses is poorly understood and will require the development of high precision actuators that are capable of stretching either isolated cells, or small colonies of cells, in combination with high-resolution detection methodologies.

In this paper, we present the conceptual design of arrays of single cell sized actuators able to apply $10-20 \%$ uni-axial strain to individual cells. A systematic Finite Element Analysis (FEA) is then employed to study behavior of the device and predict the achievable strain at various voltages and geometry parameters enabling us to find the optimized parameters. Finally the fabrication process of the first device is illustrated.

\section{CONCEPTUAL DESIGN}

The device is fabricated by patterning compliant gold ion implanted electrodes on both sides of a $30 \mu \mathrm{m}$ thick PDMS film, which is bonded onto a stiff PDMS or other rigid substrate containing trenches (Figure 5). The top of the membrane is a continuous implanted electrode and the bottom of the membrane has only narrow implanted electrodes. The active part of the device is where the implanted lines are suspended over a trench: at all these intersections, the elastomer can be electrostatically compressed, leading to in-plane strain, as shown in an exaggerated manner in top of figure. While the electrostatic force is present between the top "ground plane" and the long bottom wires, since the PDMS is bonded to the substrate, it can only expand over the trenches. The size of the actuator is thus defined by the width of the trenches and the width of the implanted bottom electrodes. 


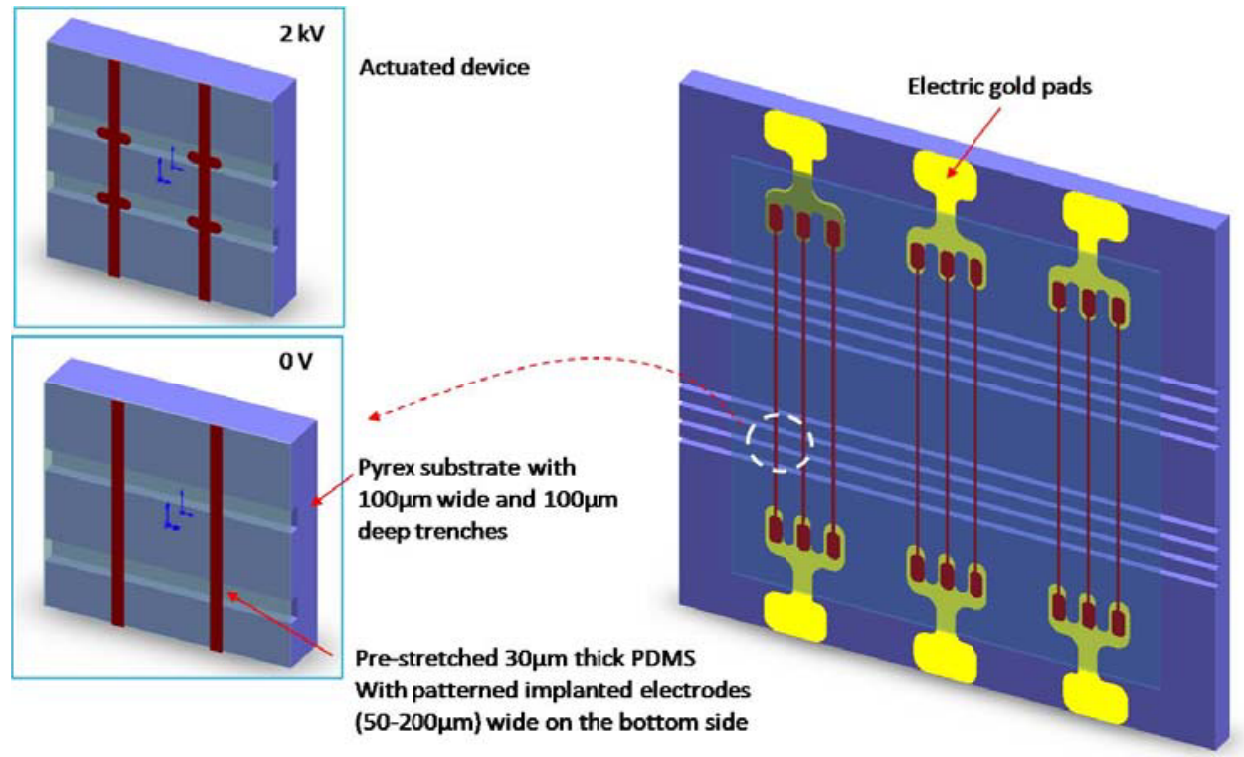

Figure 5- Simplified schematic of the cell stretching chip. Red vertical lines are $100 \mu \mathrm{m}$ wide ion-implanted electrodes on the bottom side of a $30 \mu \mathrm{m}$ thick PDMS membrane. Horizontal lines are $100 \mu \mathrm{m}$ deep $100 \mu \mathrm{m}$ wide trenches in the substrate. The membrane is bonded to the rigid substrate, except over the trenches. There is a continuous (not visible here) implanted electrode on top of the membrane. The intersection of implanted line and trench forms individual EAP actuators, $100 \times 100 \mu \mathrm{m}^{2}$, onto which cells attach following a patterned surface treatment.

The cells will be attached to each actuator following a patterned surface treatment and can then be stretched with the PDMS actuator as depicted in Figure 6.

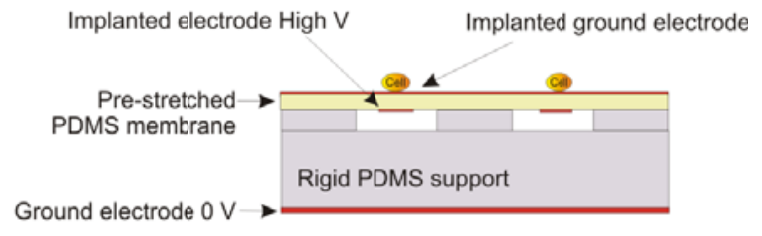

a)

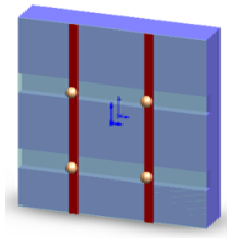

ov

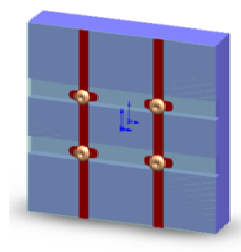

$2 \mathrm{kV}$

b)

Figure 6- left: cross-section showing two cells on two actuators. right: device with 4 actuators (and 4 cells) at $0 \mathrm{~V}$ (left), and when actuated (right), showing in an exaggerate way the strain of the implanted region (and hence the cell) which spreads along the channel.

\section{FINITE ELEMENT SIMULATION}

Finite element method is employed to model and study the behavior of the cell stretcher device, the achievable strain and the critical voltage to avoid buckling. The effect of different geometry parameters is also reviewed in order to find out the optimized design.

\section{Modeling:}

We have modeled one actuator of the arrays of uni-axial cell stretchers as shown in Figure 7. The red line represents 100 $\mu \mathrm{m}$ wide gold ion implanted wire, where the Maxwell pressure is applied in conformity with the applied voltage. The PDMS membrane is suspended over the $100 \mu \mathrm{m}$ wide trench and its bottom layer is fixed to the substrate elsewhere. 
Since our device is designed to operate in low strain levels, it is acceptable to assume the elastomer as a linear material obeying the Hook law with Poisson ratio of .495 and Young's modulus of $1 \mathrm{MPa}$.

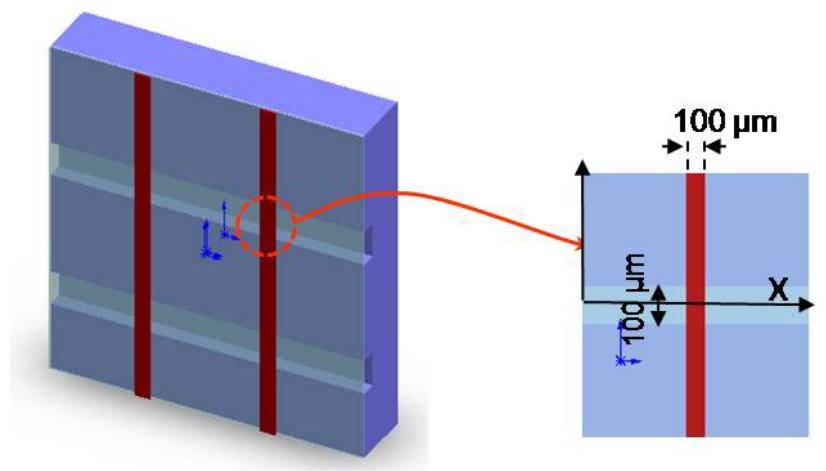

Figure 7- One cell of the arrays of micro-actuators is modeled. The red vertical line represents the $100 \mu \mathrm{m}$ wide wire of ion implanted electrode and the light blue horizontal line is the $100 \mu \mathrm{m}$ wide channel on the substrate over which the PDMS is suspended.

When $2.5 \mathrm{kV}$ voltage is applied to the electrodes, the electrostatic attraction between the opposite charges on opposing electrode generate stress on the film causing it to contract in thickness and expand in area. The expansion of area occurs along the trenches where elastomer is free to move. Deformation of the elastomer along the centerline of the channel is plotted in Figure 8, where it is shown that deformation is zero at the center and is increased symmetrically and linearly from the center of channel toward the edges to about $5 \mu \mathrm{m}$. The linear deformation of the elastomer also results in a constant $\mathrm{X}$-axis strain of about $10 \%$ on the actuator (Figure 9 ).
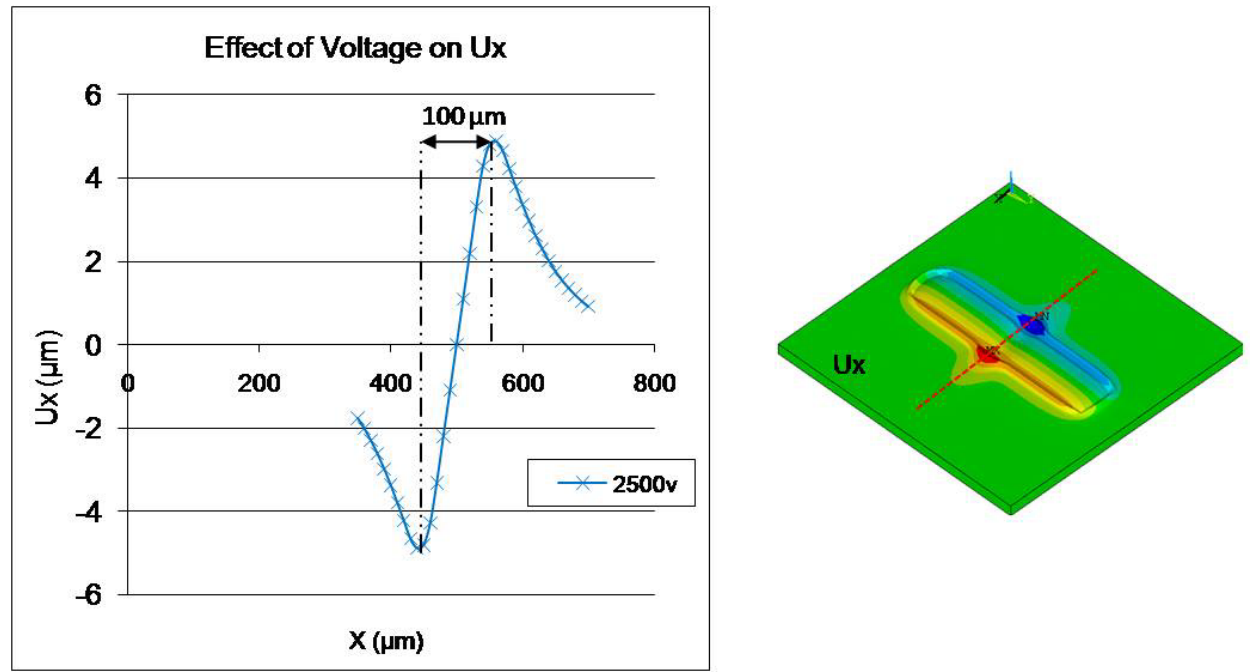

Figure 8- Deformation of the membrane along the channel, increases linearly from the center toward the edges, for $2.5 \mathrm{kV}$ voltage difference, $100 \mu \mathrm{m}$ wide wire and $100 \mu \mathrm{m}$ wide trench, with no pre-stretch of the membrane. 

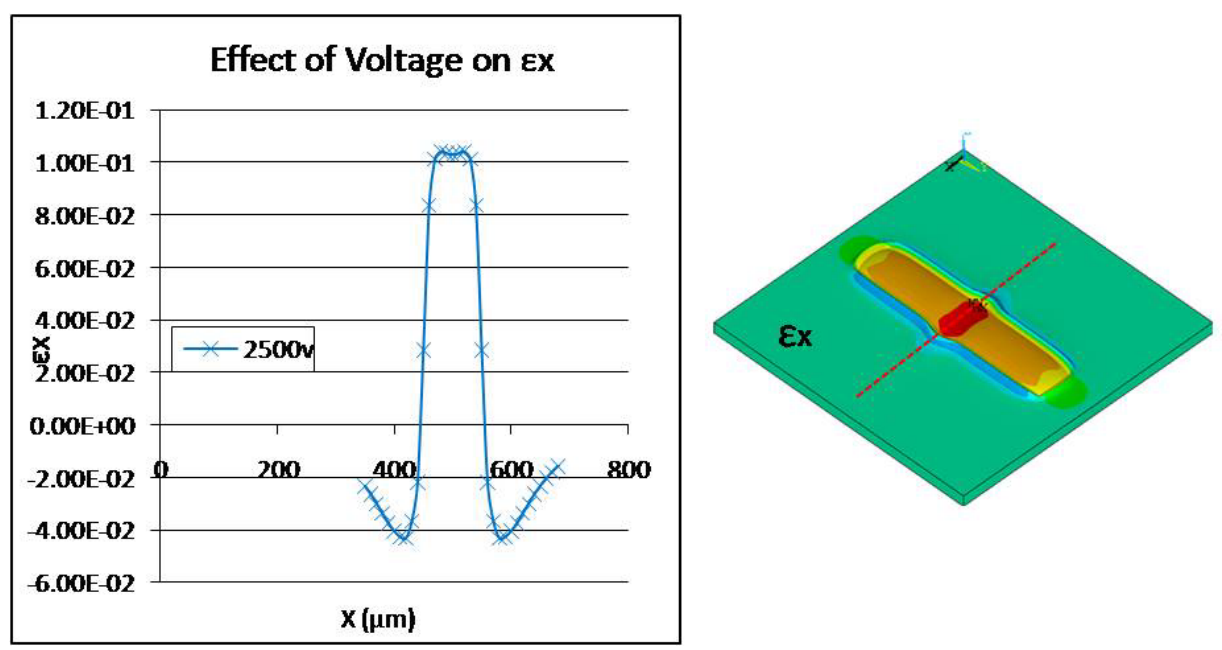

Figure 9- Strain profile along the trench, which indicates constant strain on the actuator, same conditions as Figure 8.

The achievable strain is strongly dependant to the applied voltage to the electrodes. As indicated in Figure 10 a nonstretched membrane can reach strain of $20 \%$ at $3.5 \mathrm{kV}$. But it should be considered that the maximum applicable voltage is limited by the break down voltage of PDMS, pull in instability of the elastomer and also the buckling voltage. With our fabrication process we usually can increase the voltage up to $3 \mathrm{kV}$ to a $30 \mu \mathrm{m}$ PDMS membrane before reaching the material failure.

Since the device is designed to operate in uni-axial stretching mode, the buckling voltage was also simulated. A nonlinear buckling analysis ended to $4.2 \mathrm{kV}$ for the first mode of mechanical instability, which is much higher than the operating range of our device.

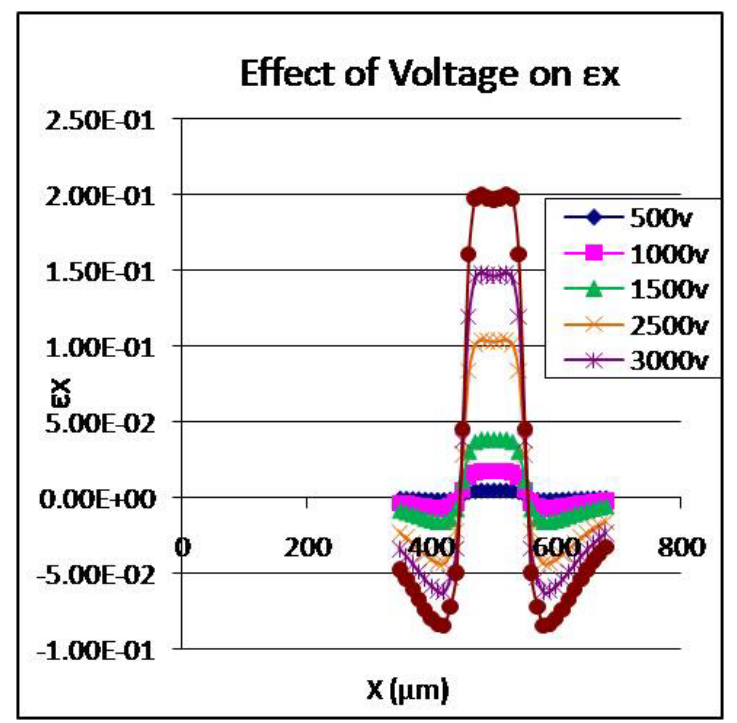

Figure 10- achievable x-axis strain on a $100 \mu \mathrm{m} \times 100 \mu \mathrm{m}$ actuator can be increased up to $15 \%$ at $3 \mathrm{kV}$ for a nonstretched, $30 \mu \mathrm{m}$ membrane.

One of the other factors that significantly influence the strain is pre-stretching the elastomer before bonding it to the substrate e.g. $30 \%$ pre-stretch can enhance the strain about $17 \%$ at $2 \mathrm{kV}$ (see Figure 11). 
Strain in Dielectric Elastomer Actuators (DEA) is also dependant to the initial thickness. As explained in the introduction, the equivalent electrostatic stress in DEAs scales as the inverse second power of thickness and so does the strain due to the Hook law, see equation 1.

$$
\sigma=-\varepsilon_{0} \varepsilon_{r} \frac{V^{2}}{z^{2}}=Y . s_{z} \Rightarrow s_{z}=-\frac{\varepsilon_{0} \varepsilon_{r}}{Y} \frac{V^{2}}{z^{2}}
$$

where $\sigma$ is the stress, $\varepsilon_{r}$ is the relative permittivity, $Y$ is the Young's modulus, $z$ is the initial thickness and $S_{x}$ and $S_{z}$ represent the strain in $\mathrm{x}$ and $\mathrm{z}$ directions.

It is seen in Figure 11 that strain in $\mathrm{x}$ direction increases significantly when the thickness is decreased. One should note that thin membranes buckle more readily under compressive in-plane loads from the electrostatic pressure and the reaction forces from fixed boundaries. Pre-stretching can increase the buckling voltage, e.g., for a $100 \mu \mathrm{m} \times 100 \mu \mathrm{m}$ micro-actuator with a $30 \mu \mathrm{m}$ thick membrane, the buckling voltage increases from $4.2 \mathrm{kV}$ to $4.5 \mathrm{kV}$ when the membrane is initially stretched by $20 \%$. Moreover, due to the breakdown strength of elastomer, the maximum applicable voltage will also be decreased with thinning the membrane.
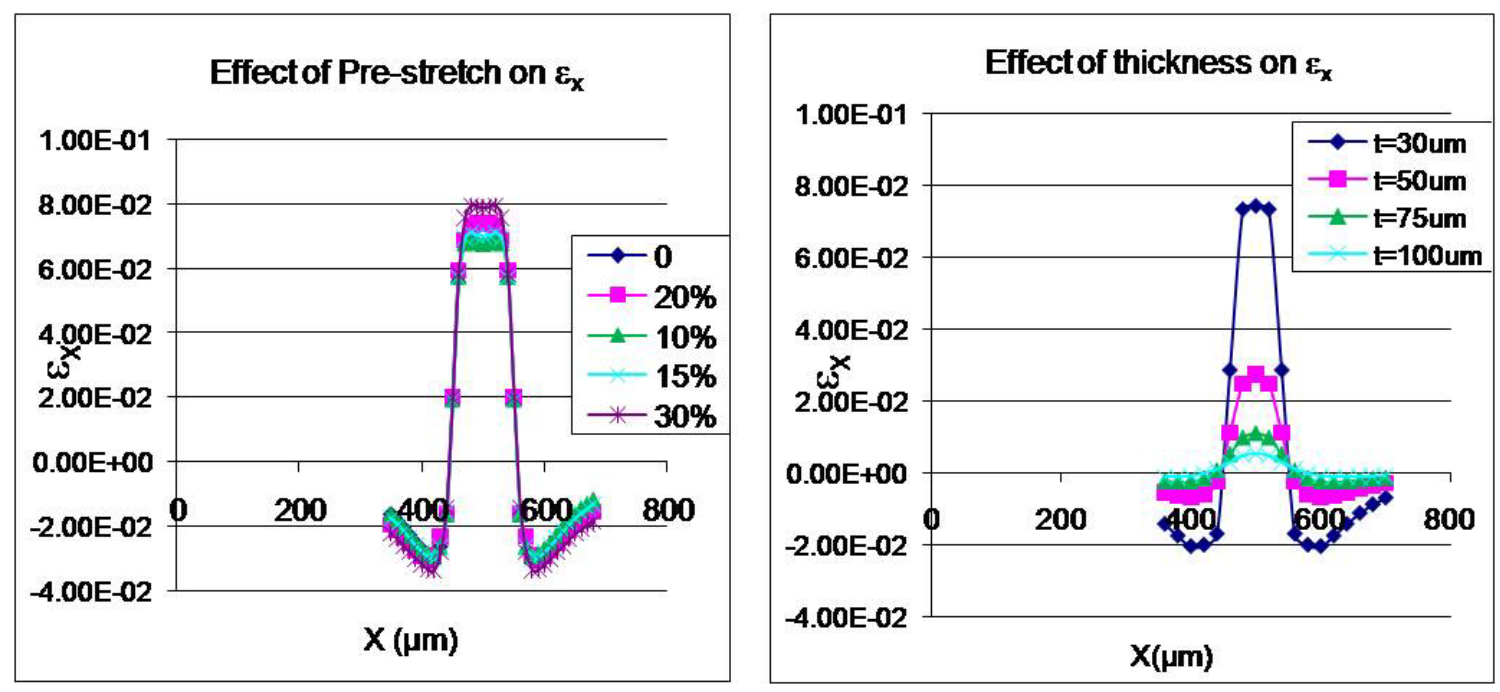

Figure 11- Effect of pre-stretching the elastomer and of the initial thickness on achievable x-axis strain of the actuator for $2 \mathrm{kV}$ voltage difference, $100 \mu \mathrm{m}$ wide wire and $100 \mu \mathrm{m}$ wide trench.

Each dielectric elastomer actuator (DEA) in our cell stretcher device is a rectangular thin elastomer, fixed at two boundaries and free to move at the other two. Therefore, in addition to the thickness, voltage and pre-stretch, which are the three important parameters for all DEAs, the device geometry (i.e., the length and width of the rectangular actuator, which for our case are the width of trenches in the substrate and the width of implanted wires, respectively) have an influence on the deformation of the elastomer.

Decreasing width of implanted electrodes will decrease the actuator area and hence reduce the electrostatic force, leading to less deflection. However the strain, which is the gradient of displacement in $\mathrm{x}$ direction $\left(\frac{\partial u}{\partial x}\right)$, is increased for narrower electrodes due to decrease of $\Delta x$. Increasing the width of trenches increases the displacement and hence strain. Reviewing the effect of different parameters on the performance of the micro-actuator, we can define the optimized design which leads to $10-20 \%$ uni-axial strain at lower voltages, avoiding any potential breakdown issues. This optimized device is made with a thin membrane (30 $\mu \mathrm{m}$ or less), which is initially pre-stretched by over $30 \%$ and has compliant electrodes of width of less than $100 \mu \mathrm{m}$. The optimal width of the channels in the rigid substrate is $200 \mu \mathrm{m}$. With our current fabrication process, we cannot pattern compliant electrodes narrower than $100 \mu \mathrm{m}$, though we are working on a process to allow this. Therefore, in making the first devices, we chose the closest possible value to the optimized parameters. 

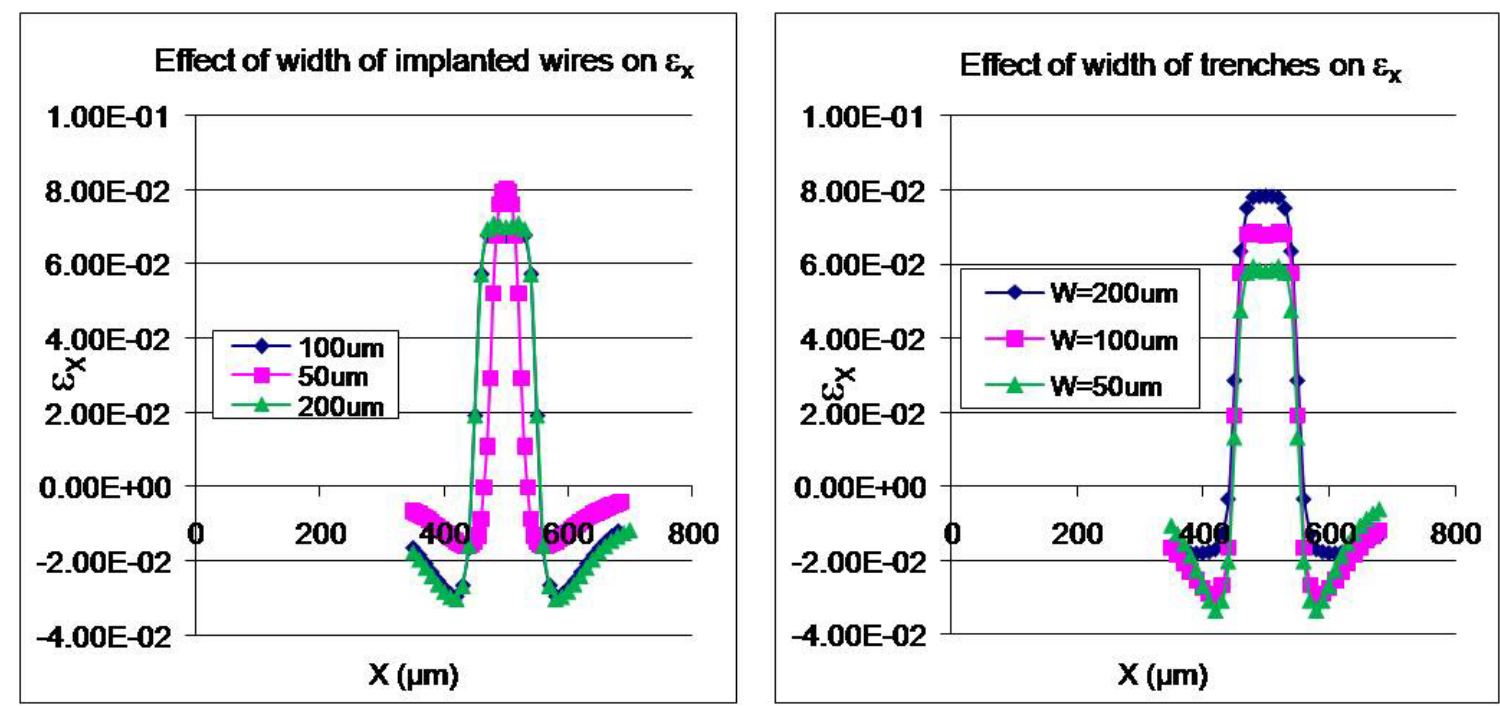

Figure 12- Effect of size of the implanted wires and width of trenches on the $\mathrm{x}$-axis strain for $2 \mathrm{kV}$ voltage difference, $30 \mu \mathrm{m}$ thick membrane and zero initial strain.

\section{FABRICATION}

The fabrication process consists of making thin layer of PDMS, patterning the compliant electrodes on both sides by ion implantation, and bonding the membranes to a stiffer substrate with trenches.

Sylgard 186 from Dow Corning is chosen as the elastomer our DEA application. It is prepared by mixing the PDMS with the curing agent with a $10: 1$ weight ratio, according to the manufacturer's indications. The mixture is then diluted with isooctane (PDMS : Solvent 10:9 weight) in order to lower its viscosity and spin-coated on the PR-coated polyamide substrate to obtain a PDMS thickness in the $20-30 \mu \mathrm{m}$ range. The PDMS is cured at $70^{\circ} \mathrm{C}$ for 3 hours. Then gold ions are implanted into the PDMS to serve as the compliant electrode on the bottom side of the elastomer, as described in detail in [[5]] and [[2]]. To contact the implanted wires, gold contacts are sputtered through a shadow mask, and wires are glued to the gold pads with conductive paste.

The substrate with trenches is made by molding a stiffer PDMS such as Dow Corning Sylgard 184 on SU-8. The gold contacts on PDMS substrates are sputtered using a shadow mask. After bonding the membrane to the substrate following 15 s plasma oxygen exposure, the gold electrodes will serve as conduction path between the backside implanted zone and the chip's border for wire connection (see Figure 13). The thin membrane is released from the polyamide holder by dissolving the sacrificial layer. Finally the top of the membrane can be ion implanted to make the top electrodes.

1)

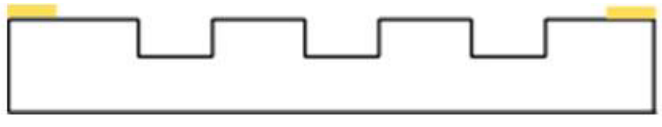

2)

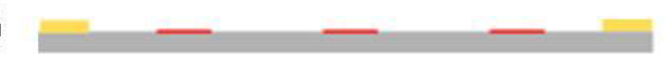

3)

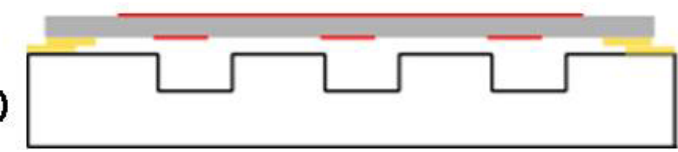

Figure 13- Fabrication process; 1) a 2mm thick PDMS (Sylgard 184) substrate is molded on a SU-8 wafer with $100 \mu \mathrm{m}$ features and after curing, gold electrodes for wire connection is made by sputtering . 2) A membrane of PDMS (Sylgard 186) is prepared by spinning and $100 \mu \mathrm{m}$ wide compliant electrodes are defined by implanting gold ions. 3) The membrane is bonded to the substrate following oxygen plasma exposure and the compliant electrode on the top side of the membrane is then implanted.

The fabricated device is shown in Figure 14, where four groups of micro-actuators can be seen that can be actuated by different voltages and frequencies make it possible to stimulate different groups of cells by various strains in the same cell culture. The device shown in Figure 14 was actuated up to $3 \mathrm{kV}$, and was observed to move. Detailed characterization is ongoing. 

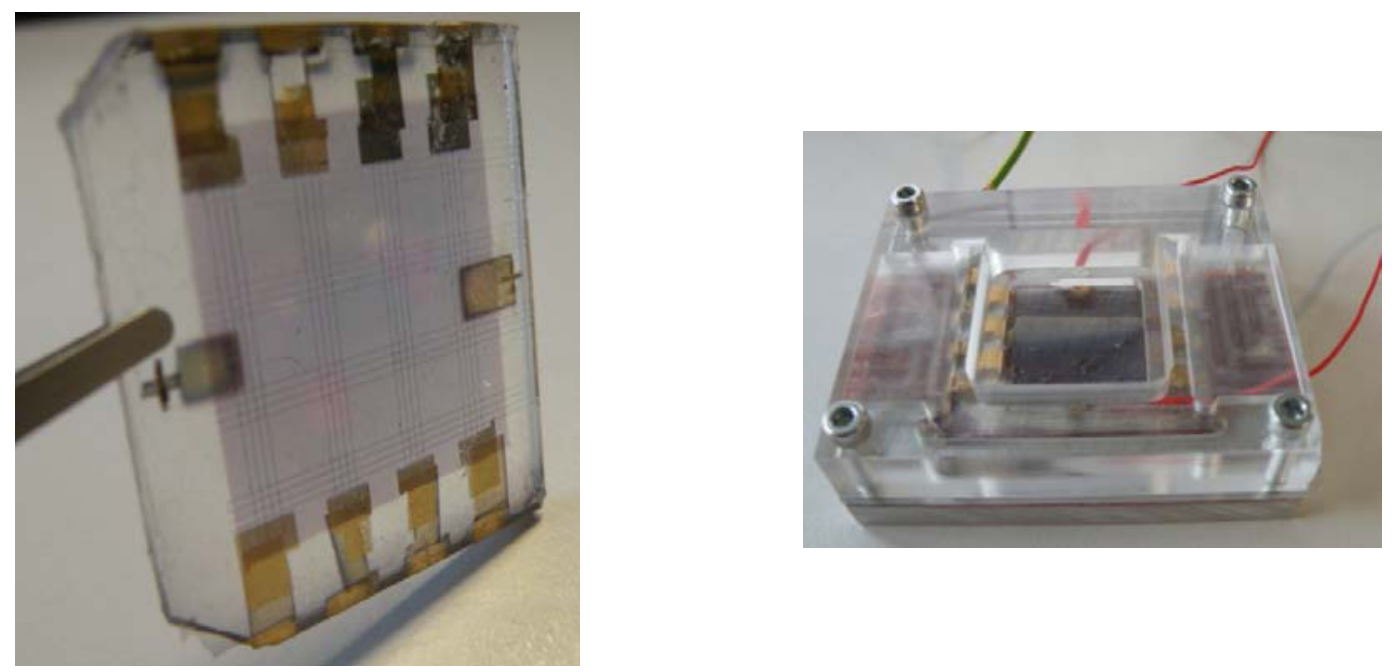

Figure 14- left; the device on made of a $30 \mu \mathrm{m}$ PDMS membrane bonded to a stiffer PDMS membrane with 100 $\mu \mathrm{m}$ trenches. Right; device with the holder, designed to allow handling with a cell culture medium and operation in an incubator.

\section{CONCLUSION}

We have shown the design, FEM optimization, and fabrication of an array of EAP devices designed to apply over $10 \%$ uniaxial mechanical strain to single cells in a larger culture. The finite element simulation showed that the device can apply the required strain at the reasonable voltages, well below the break-down voltage, pull-in instability and buckling voltage. The effect of different design parameters on the performance of the device was reviewed to obtain the optimized design to achieve the required strain at lower voltages. The fabrication process of the device is then explained which leads to a device with four groups of micro-actuators that can be actuated by different voltages and frequencies, make it possible to stimulate groups of cells differently on the same cell culture. Future work focuses on electro-mechanical characterization, testing with cells, and improved fabrication sequences to ensure compatibility with standard inverted microscopes.

\section{ACKNOWLEDGEMENTS}

We acknowledge financial support from the Swiss National Science Foundation grant \#20020-120164, and thank Dr. A. Franco-Obregon, E. Thomasson, and Prof J. Vörös from the ETHZ-LBB for helpful advice.

\section{REFERENCES}

[1] Brochu, P., Pei, Q., "Advances In Dielectric Elastomers For Actuators And Artificial Muscles”, Macromolecular. Rapid Communications, 31 (1), 10-36, (2010).

[2] Rosset, S., Niklaus, M., Dubois, P., Shea, H. R., "Large-Stroke Dielectric Elastomer Actuators With Ion- Implanted Electrodes", Journal of Microelectromechanical Systems, 18 (6), 1300-1308, (2009).

[3] Rosset, S., Niklaus, M., Dubois, P., Shea, H. R., "Mechanical characterization of a dielectric elastomer microactuator with ion-implanted electrodes", Sensors and Actuators A: Physical, 144 (1), 185-193, (2008).

[4] Dubois, P., Rosset, S., Koster, S., Stauffer, J., et al, "Microactuators based on ion implanted dielectric electroactive polymer (EAP) membranes", Sensors and actuators A: Physical, 130-131, 147-154, (2006). 
[5] Rosset, S., Niklaus, M., Dubois, P., Shea, H. R., "Metal Ion Implantation for the Fabrication of Stretchable Electrodes on Elastomers", Advanced Functional Materials, 19, 470 - 478, (2009).

[6] Rosset, S., Niklaus, M., Dubois, P., Dadras, M., Shea, H. R., "Mechanical properties of electroactive polymer microactuators with ion-implanted electrodes", Proc. SPIE, 6524, 10-1-10-11, (2007).

[7] Brown, I. G., "Vacuum arc metal plasma production and the transition of processing mode from metal ion beam to dc metal plasma immersion", Surface and Coatings Technology, 136 (1-3), 16-22, (2001).

[8] Niklaus, M., Rosset, S., Dadras, M., Dubois, P., Shea, H. R., "Microstructure of 5 keV gold-implanted polydimethylsiloxane", Scripta Materialia, 59, 893-896, (2008).

[9] Collingsworth, A. M., Torgan, C. E., Nagda, S. N., Rajalingam, R. J., Kraus, W. E., Truskey, G. A., “Orientation and length of mammalian skeletal myocytes in response to a unidirectional stretch", Cell Tissue Res, 302, 243-51, (2000).

[10] Clark, C. B., Burkholder, T. J, Frangos, J. A., "Uniaxial strain system to investigate strain rate regulation in vitro", Rev Sci Instrum, 72, 2415-23 (2001).

[11] Standley, P. R., Camaratta, A., Nolan, B. P., Purgason, C. T., Stanley, M, A., "Cyclic stretch induces vascular smooth muscle cell alignment via NO signaling”, Am J Physiol Heart Circ Physiol, 283, H1907-14, (2002).

[12] Sharma, R.V., Chapleau, M.W., Hajduczok, G., Wachtel, R.E., Waite L.J., Bhalla, R.C., Abboud, F.M., "Mechanical stimulation increases intracellular calcium concentration in nodose sensory neurons", Neuroscience, 66 (2), 433-441, (1995).

[13] Birukov, K. G., Stretch affects phenotype and proliferation of vascular smooth muscle cells. Molecular and Cellular Biochemistry 144, 131-139, (1995).

[14] Song, G., Ju, Y., Soyama, H., Ohashi, T., Sato, M., "Regulation of Cyclic Longitudinal Mechanical Stretch on Proliferation of Human Bone Marrow Mesenchymal Stem Cells", MCB, 090 (1), 1-10, (2008).

[15] Brown, T., “Techniques for Mechanical Stimulation Of Cells In Vitro: A Review”, Journal of Biomechanics, 33, 3$14,(2000)$. 